\title{
AN EXTENSIVE PRECIPITATION OF CALCIUM IN SUBCUTANEOUS TISSUE IN PATIENT WITH JUVENILE DERMATOMYOSITIS
}

\author{
Boskovic Olivera1, Borozan Sanja12 ${ }^{2}$, Vujosevic Snezana1 ${ }^{2}$, Kavaric Sreten', Djogo Aleksandar', Kazic Koviljka1, Medenica Sanja1 2 \\ 1 Internal medicine Clinic, Clinical Center of Montenegro, Podgorica, Montenegro \\ 2 Faculty of Medicine, University of Montenegro \\ Correspondence to olivbos@t-com.me; sanjaradoman@yahoo.com
}

\section{INTRODUCTION}

Calcinosis cutis reffers to a group of disorders in which calcium deposits are being formed in the skin. Depending on the pathophysiologic mechanisms, calcinosis cutis has been classified as metastatic, dystrophic, idiopathic, or iatrogenic (1). In connective tissue diseases, calcinosis is mostly of the dystrophic type and it seems to be a localized process rather than an imbalance of calcium homeostasis (2).

Juvenile dermatomyositis (JDM) is a systemic, autoimmune inflammatory muscle disorder and a small vessel vasculopathy that affects children younger than 18 years, primarily the skin and the skeletal muscles (3). Calcinosis cutis occurs in $20-40 \%$ of patients with JDM (4). Some severe cases may be associated with advanced calcinosis but the exact mechanism that leads to their development and spreading remains unclear.

\section{CASE REPORT}

A female patient firstly admitted to the hospital at the age of 17 for skin rash, malaise and pain in peripheral joints and muscles. After evaluation a syndroma overlap with dominant atypic dermatomyositis (without muscle enzymes elevated) and systemic lupus erythematosus was suspected and treatment with prednisolone and choroquine initiated.

After six months subcutaneous calcium deposits in form of multiple firm nodules appeared, firstly in gluteal region, than polytopically. The nodules were gradually enlarging, causing pain and exudation of chalky white material without a previous traumatic event. No metabolic disorders were observed; levels of PTH, phosphorus and calcium have always been in reference range; ANA and anti-dsDNA occasionally elevated.

During the last 12 years, systemic corticosteroids and other different immunosuppressive and immunomodulatory agents (methotrexate, cyclophosphamide, azathioprin, mycophenolat, colchicine), intravenous bisfosfonates, imunoglobulins and calcium antagonists were given in attempt to establish a better disease control, stop spreading and reduce a calcinosis along with tissue damage but without any significant improvement.
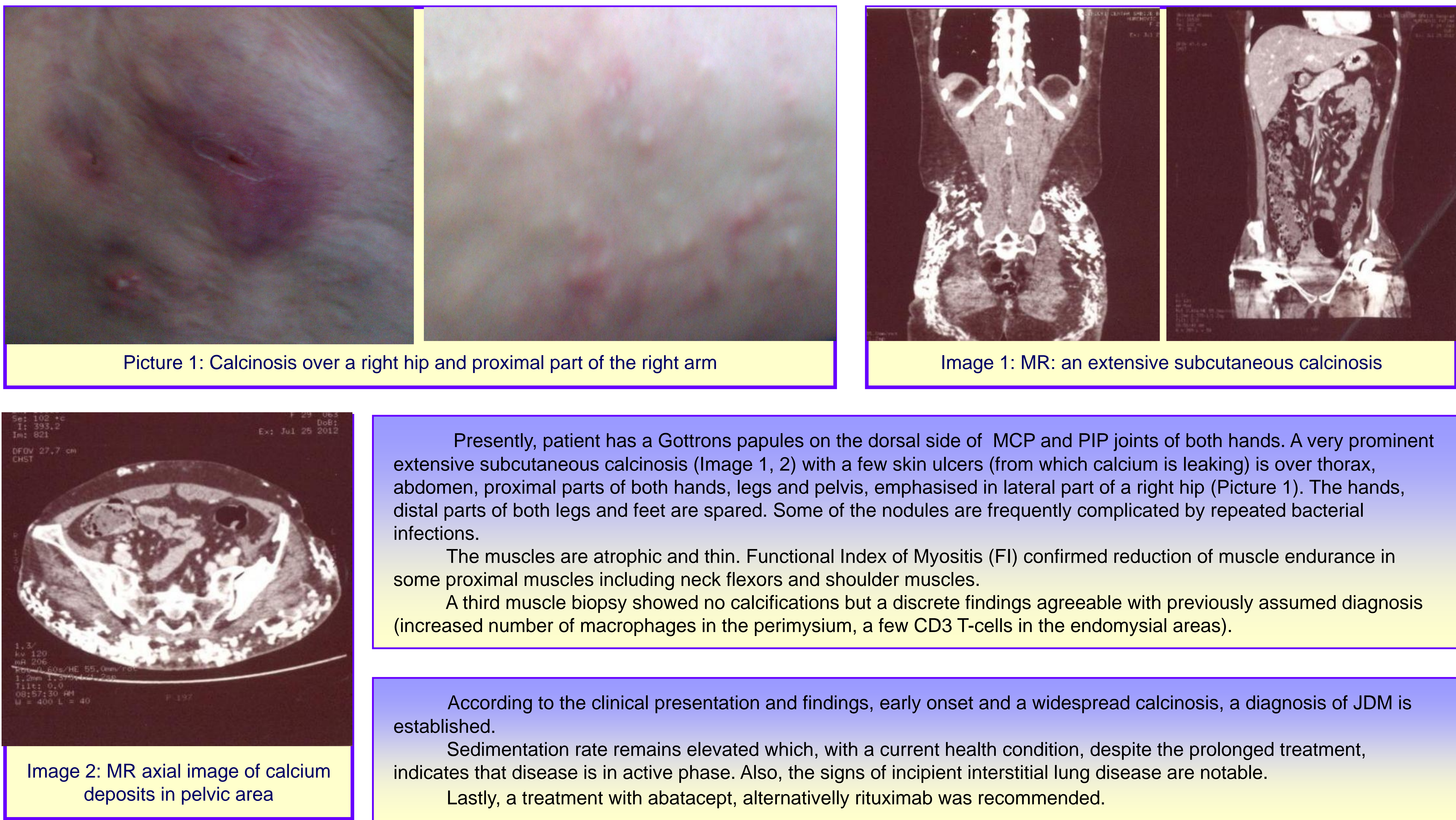

Presently, patient has a Gottrons papules on the dorsal side of MCP and PIP joints of both hands. A very prominent extensive subcutaneous calcinosis (Image 1,2) with a few skin ulcers (from which calcium is leaking) is over thorax, abdomen, proximal parts of both hands, legs and pelvis, emphasised in lateral part of a right hip (Picture 1). The hands, distal parts of both legs and feet are spared. Some of the nodules are frequently complicated by repeated bacterial infections.

The muscles are atrophic and thin. Functional Index of Myositis $(\mathrm{FI})$ confirmed reduction of muscle endurance in some proximal muscles including neck flexors and shoulder muscles.

A third muscle biopsy showed no calcifications but a discrete findings agreeable with previously assumed diagnosis (increased number of macrophages in the perimysium, a few CD3 T-cells in the endomysial areas).

According to the clinical presentation and findings, early onset and a widespread calcinosis, a diagnosis of JDM is established.

Sedimentation rate remains elevated which, with a current health condition, despite the prolonged treatment,

indicates that disease is in active phase. Also, the signs of incipient interstitial lung disease are notable.

Lastly, a treatment with abatacept, alternativelly rituximab was recommended.

\section{CONCLUSION}

Althought a number of drugs are often given for the treatment of calcinosis, the approach to menagement is still not established due to inconsistent responses. Revealing a patogenesis of calcium deposits would certanly bring a new insight to this and similares cases.

\section{References}

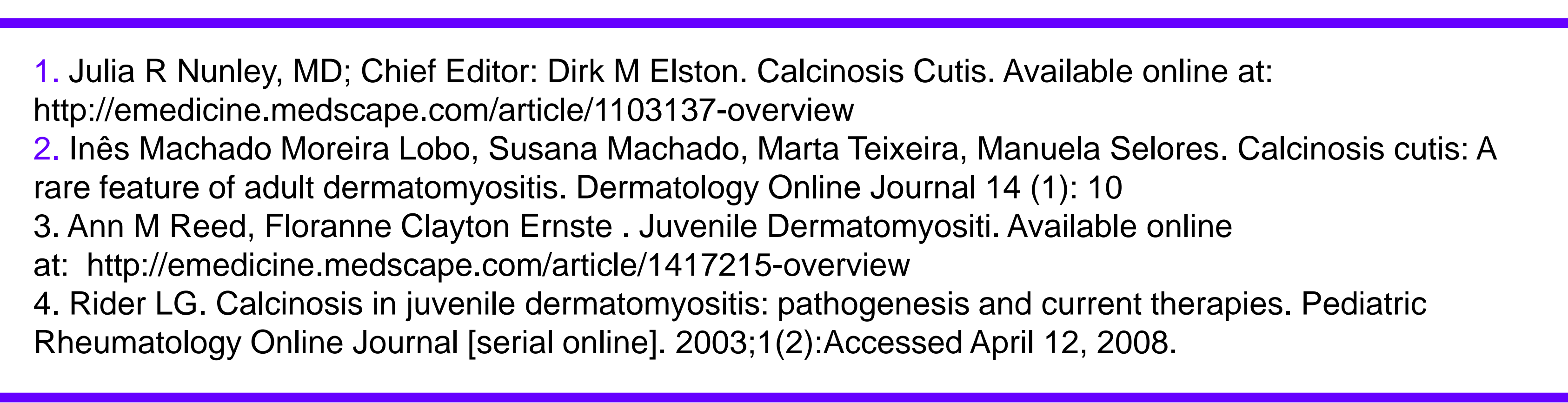

